\title{
Rim calcification in primary liver cell carcinoma mimicking hydatid disease
}

\author{
A W Mitchell, J N P Higgins, J Tate, R Dick
}

\begin{abstract}
The case described is of a primary liver cell carcinoma (PLCC), which was initially misdiagnosed as hepatic hydatid disease. The computed tomographic appearances of hepatic hydatid disease are often characteristic although not specific. When supported by the clinical findings, however, they will probably not be confused with other liver masses. In this case the presence of peripheral rim calcification, commonly seen in hydatid disease but which has not been previously noted in PLCC, lead to an erroneous diagnosis.

(Gut 1994; 35: 567-568)
\end{abstract}

Hydatid disease remains a common disease especially in central Europe and even today 'it is unwise to dismiss it as a curiosity'. ${ }^{2}$ Primary liver cell carcinoma is a comparatively rare tumour in western Europe (1-2/100000), although its incidence is greatly increased in patients with cirrhosis. This case shows the only recorded case of rim calcification in a patient with untreated primary liver cell carcinoma (PLCC). He was referred from abroad where clinical and computed tomographic findings seemed strongly suggestive of hydatid disease and treatment was planned on this basis. Radiodiagnosis and Hepatobiliary Surgery, The Royal Free

Hampstead NHS Trust, London

A W Mitchell

J N P Higgins

J Tate

R Dick

Correspondence to:

Dr A W Mitchell,

Department in

Radiodiagnosis, The Royal

Free Hampstead NHS Trust,

Pond Street, London

NW3 2QG.

Accepted for publication 26 July 1993

\section{Case report}

A 56 year old Greek plumber presented with a two month history of upper abdominal discomfort, intermittent fever, mild obstructive jaundice, and tenderness in the right upper quadrant. As part of the investigations computed tomography was performed, which showed a multicystic lesion in the liver with peripheral curvilinear calcification (Figure). A diagnosis of hydatid disease was made. Jaundice was thought to be secondary to external compression of the major bile ducts by the mass. At laparotomy a solid tumour was found in the liver and a diagnostic biopsy performed. Histological review of the lesion showed PLCC and cirrhosis with no special features. Hepatitis B serology was positive but there was no rise in the serum $\alpha$ feto protein concentration. After his recovery he was referred to the Royal Free Hospital for further management.

Investigations for metastases were negative. A second laparotomy was performed but intraperitoneal deposits were found and a planned resection was abandoned. Biopsy of a diaphragmatic lesion also seen on computed tomography confirmed PLCC. After a period of recovery the patient had a systemic course of epirubicin and returned home.

\section{Discussion}

Many causes of hepatic calcification have been described. ${ }^{3}$ The presence of calcification, however, is not usually helpful in the differential diagnosis of hepatic masses. Relating specifically to malignancy it occurs with almost equal frequency in PLCC and colonic carcinoma secondaries. $^{34}$ The computed tomographic morphology of the calcification, is only comparatively specific when it is peripheral, dense, and curvilinear as seen in the walls of hydatid cysts and amoebic abscesses. Otherwise the pattern is unhelpful. ${ }^{3}$ In the case described it was the combination of rim calcification and the demographic frequency of hydatid disease that lead to an erroneous diagnosis.

Involvement of the liver by Escherichia granulosus is suggested by computed tomography by the presence of well defined oval or spherical lesions of near water density but these signs are not specific and may be seen in abscesses, cystic
(A) Computed tomography through the right lobe of the liver. Curvilinear calcification is arrowed in the wall of the complex lesion; $(B)$ Enhanced computed tomography 1.5 cm below $(A)$. Arrows point to wall calcification. Within the large cystic space enhancing material is present, while three smaller cystic lesions are seen $3 \mathrm{~cm}$ beyond the main mass.
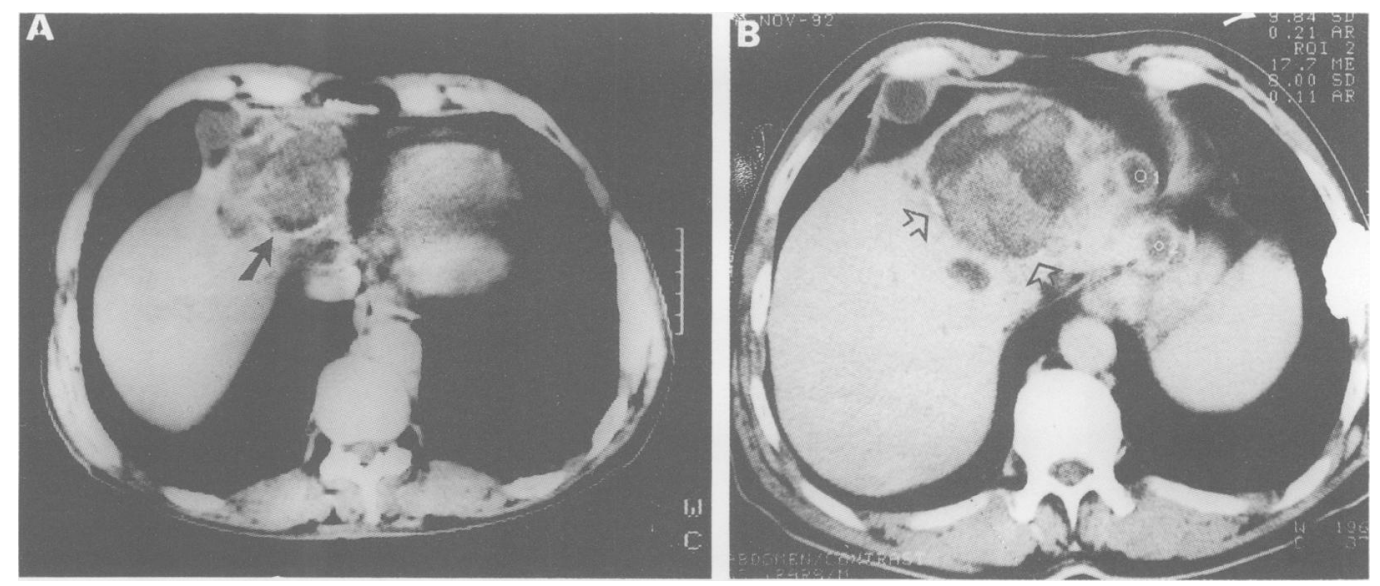
metastases, Caroli's disease or congenital cysts. ${ }^{5}$ Rim calcification occurs in $10-20 \%$ and when seen in a patient from an endemic area is strongly suggestive of hydatid disease. ${ }^{56}$ Only the finding of daughter cysts or membrane detachment, however, can be considered diagnostic. ${ }^{7}$

Escherichia alveolaris is less common than $E$ granulosis but is more probably confused with hepatic malignancy in that, larger lesions are mixed with areas of necrosis, calcification, and cyst formation. In $13 \%$ of lesions calcification may be seen in the walls of cysts and again when seen in a patient from an endemic area hydatid disease is the most probable diagnosis.

PLCC is normally isodense with liver but may be hypodense. Most lesions show considerable heterogenious enchancement after intravenous contrast. ${ }^{8}$ Morphologically they may be single or multiple, poorly or well defined lesions. Calcification occurs in up to $15 \%$ of cases; in one case minimal arcuate calcification has been seen near the periphery of a diffuse tumour after chemotherapy. ${ }^{3}$

The diagnosis of hydatid disease does not depend on the presence or absence of rim calcification. Nevertheless rim calcification is suggestive of hydatid disease and is particularly useful in the diagnosis of alveolar hydatid disease, ${ }^{9}$ which can be very difficult to differentiate from malignancy. Rim calcification, however, is not pathognomonic of hydatid and in the absence of further corroborative signs other possibilities must be considered.

In this patient attenuation values significantly higher than water were present within the lesion. This could have represented secondarily infected cysts $^{56}$ although in such cases there is often surrounding low attenuation suggesting oedema.

Nevertheless, infection by $E$ granulosus was effectively ruled out by the diffuse contrast enhancement of the area. Infection of $E$ alveolaris on the other hand, could not be excluded on this basis because contrast enhancement may occur and is therefore unhelpful in differentiating this from other hepatic masses.

In conclusion this is the first report of rim calcification in association with PLCC. Its presence in conjunction with a hepatic mass should not distract the radiologist or clinician from a careful review of the other features of an abnormality before reaching a diagnosis.

We would thank professor $\mathrm{K} \mathrm{E}$ Hobbs for his support and under whose care this patient was.

1 Radford AJ. Cestodes: hydatid disease. In: Wetherall, Ledingham and Warrell. The Oxford Textbook of Medicine. 2nd ed. Oxford: Oxford University Press, 1987: 5.561-65. 2 Matossian RM. Distribution of hydatid disease. Bull World Health Organ 1977; 55: 449.

3 Scatarige JC, Fishman EK, Sarsouk FA, Seigleman SS. Computed tomography of liver masses. $\mathcal{F}$ Comput Assist Tomogr. 1983; 7: 83-9.

4 Darlack JJ, Moskowitz M, Kattan KR. Calcifications in the liver. Radiol Clin North Am 1980; 18: 209-19.

5. Barnes PA, Thomas JL, Bernadino ME. Pitfalls in the diagnosis of hepatic cysts by computed tomography. Radiology 1981; 141: 129-33.

6 Beggs I. The radiological appearances of hydatid disease of the liver. Clin Radiol 1983; 34: 555-63.

7 Kalvovidouris A, Pissiotis C, Pontifex G, Gouliamos A, Pentea $S$, Papavassiliou C. Computed tomography of multivesicular hydatid cysts. F Comput Assist Tomogr 1986; 10: 428-31.

8 Itai Y, Araki T, Furui S, Tasaka A. Differential diagnosis of hepatic masses on computed tomography, with particular reference to hepatocellular carcinoma. I Comput Assist Tomogr 1981; 5: 834-42.

9 Choji K, Fujita N, Chen M, Spiers ASD, Morita Y, Shinohara $M$, et al. Alveolar hydatid of the liver: computed tomography and transabdominal ultrasound with histopathological correlation. Clin Radiol 1992; 46: 97-103. 